\title{
Prevalence and microbiological features of spontaneous bacterial peritonitis in hospitalized ascitic patients: Single center study
}

\begin{abstract}
Spontaneous bacterial peritonitis (SBP) is a common serious complication in cirrhotic patients. The aim of this work is to determine the microbiological causes and their susceptibility pattern in patients with SBP admitted to Hepatology Unit at Mansoura Specialized Medical Hospital. This study included 400 patients with liver cirrhosis, ascites and clinical picture suggestive of SBP. All patients suspected to paracentesis. The aspirated fluid was examined for total leucocyte count and bacterial culture. 179 patients $(58.3 \%)$ out of 400 SBP patients were found to have Culture Negative Neutrocytic Ascitis (CNNA) while were $128(41.7 \%)$ patients had positive cultures. The causative microorganism was found to be $E$ coli which was found in 57 patients (44\%), staph. aureus in 32 patients $(25 \%)$ and then K pneuminiae found in 20 patients (16\%) finally strept pneumoniae in 19 patients (15\%) of culture positive patients. Amoxicillin and Clavulanic acid (AMC) were the most sensitive antibiotic to be used as an oral antibiotics giving $92 \%$ sensitivity on all the detected bacteria.
\end{abstract}

Keywords: SBP, CNNA, strept pneumonia, HCV, HBV, asterixis
Volume 6 Issue 2 - 2018

Sahar El Gharabawy,' Noha EL Mashad, ${ }^{2}$ Tarek Fouad Sheta,' Ibrahim Elsayed Ibrahim Abdel Aziz ${ }^{3}$

'Department of internal medicine, Faculty of medicine Mansoura University, Egypt

${ }^{2}$ Department of clinical pathology, Faculty of medicine Mansoura University, Egypt

${ }^{3}$ Aga Hospital, Pakistan

Correspondence: Tarek Fouad Sheta, Department of internal medicine, Faculty of medicine, Mansoura University, Egypt, Emaildrtarek_sheta@yahoo.com

Received: March 20, 2018| Published: April 26, 2018

\section{Introduction}

Spontaneous bacterial peritonitis (SBP) is infection of the ascitic fluid that occurs in the absence of a visceral perforation or abdominal inflammatory focus such as abscess, acute pancreatitis. SBP is a serious complication in cirrhotic patients. ${ }^{1,2}$

The prevalence of SBP on a study was conducted on 100 cirrhotic,ascitic patients presented to the Internal Medicine Department, Kasr Elaini hospital was about $57 \% .{ }^{3}$ It has been suggested that the microbiological causes of SBP and the susceptibility of the causative organisms to antibiotics are changing for several reasons. ${ }^{4,5}$ We are in need of these results to treat patients successfully to prevent complications.

\section{Patients \& methods}

This study included 400 patients (222 were males and 178 were females) admitted to Hepatology Unit at Mansoura Specialized Medical Hospital with liver cirrhosis, ascites and clinical picture suggestive of spontaneous bacterial peritonitis (abdominal pain, fever and or impaired level of consciousness). This study was performed during the period between 11-2014 and 2-2015.

Depending upon polymorph leucocytic count in the aspirated ascitic fluid, patients were divided into two groups:

A-SBP patients with ascetic fluid polymorph nuclear count $(\mathrm{PMNL}) \geq 250$ cells $/ \mathrm{mm}^{3}$

B-NON SBP with ascetic fluid polymorph nuclear count (PMNL) $<250$ cells $/ \mathrm{mm}^{3}$

Patients who were on antibiotic therapy were excluded.

\section{Methods}

\section{All patients were submitted for}

I. History taking ( $\mathrm{HCV}, \mathrm{HBV}$, alcohol consumption, previous attacks of spontaneous bacterial peritonitis).

II. Clinical examination including symptoms: abdominal distension, elevation of body temperature, abdominal pain, impairment of consciousness level, vomiting of blood and bleeding per rectum and signs: ascites, fever, abdominal tenderness, encephalopathy \{flabbing tremors (asterixis) impaired consciousness level and confusion , jaundice and lower limb oedema.

III. Laboratory investigation

On the day of admission, all patients suspected to paracentesis under local anaethestic and strict aseptic condition was done.

The aspirated fluid was examined for

Total leucocyte count

Culture on automated blood culture system (Bact/ALERT 3D) from biomerieux.

\section{Statistical analysis}

Data were tabulated, coded then analyzed using the computer program SPSS (Statistical package for social science) version 17.0.

\section{Results}

The study showed a significant increase in gram negative bacteria as a causative micro-organism in culture positive SBP patients. The causative organisms detected were E. coli (44\%), Staph. aureus (25\%), K. pneumoniae (16\%) and Strept. pneumoniae (15\%). 
The culture results showed that $E$ coli was highly sensitive to Amoxicillin and clavulanic (91.2\%) followed by erythromycin $(89.4 \%)$ and amoxicillin (87.7\%). Staph aureus was most sensitive to cefoprazone $(100 \%)$ while $k$. pneumoniae is most sensitive to meropenem (95\%) It also shows that Strept pneumoniae is most sensitive to AMC, Ofloxacin and meropenem (100\%).

\section{Discussion}

Spontaneous bacterial peritonitis is a grave and fatal complication for individuals with liver cirrhosis and ascites with high mortality and recurrence rates and poor long term prognosis. ${ }^{6}$ In this study, we found that $78.5 \%$ of SBP patients were in class C while $21.5 \%$ in class B according to child Pugh classification (Table 1) (Table 2) (p value $<0.001)$

Table I Classification of all patients according to child pugh score

\begin{tabular}{lllllll}
\hline & \multicolumn{3}{c}{ SBP(307) } & \multicolumn{4}{c}{ Non SBP(93) } & \multirow{2}{*}{ P } \\
\cline { 2 - 6 } & & No & $\%$ & No & $\%$ & \\
\hline Childpuogh & B & 66 & $21.50 \%$ & 42 & $45.20 \%$ & $<0.001 * *$ \\
& C & 241 & $78.50 \%$ & 51 & $54.80 \%$ & \\
\hline
\end{tabular}

In this study that was conducted on patients having decompensated cirrhosis, we found a high prevalence of SBP in 307 patients $(76.7 \%)$ (Table 3).

This agrees with a study by Abeer et al. ${ }^{3}$ who found the prevalence of SBP in a study was conducted on 100 cirrhotic, ascetic patients presented to the Internal Medicine Department, Kasr Elaini hospital was about $57 \%$.

In another study by Gills et al. ${ }^{7}$ it was found that the prevalence of SBP in ascitic patients who were admitted into the medical ward of the Ekiti State University Teaching Hospital (EKSUTH), AdoEkiti, Nigeria is about $50 \%-60 \%$.

According to results of ascetic fluid culture of SBP patients, it was also found that 179 patients (58.3\%) among 400 SBP patients were found to have Culture Negative Neutrocytic Ascitis (CNNA) while were $128(41.7 \%)$ patients had positive cultures (Table 4$)$.

Payal et al. ${ }^{8}$ studied 217 clinically suspected cases of SBP and concluded that $71(43.80 \%)$ had ascitic fluid polymorphonuclear cells (PMN) count $\geq 250 / \mathrm{mm}^{3} .31(43.6 \%)$ cases were culture positive and $40(56.4 \%)$ cases were culture-negative neutrocytic ascites.

Table 2 Laboratory data

\begin{tabular}{llllll}
\hline & SBP (307) & & Non SBP (93) & P \\
\hline WBC & 8.7 & $1.30-32.00$ & 9 & $4.10-25.30$ & 0.9 \\
HB & 9.2 & \pm 1.98 & 9.28 & \pm 2.04 & 0.7 \\
Platlets & 72 & $8.80-400.00$ & 81 & $12.00-200.00$ & 0.6 \\
Creatinine & 1.8 & $0.70-7.00$ & 1.7 & $0.70-7.00$ & 0.4 \\
Albumin & 2.68 & \pm .77 & 2.79 & \pm .71 & 0.2 \\
HCVab & 142 & $46.30 \%$ & 38 & $40.90 \%$ & 0.36 \\
HBVsAg & 28 & $9.10 \%$ & 13 & $14.00 \%$ & 0.17 \\
AST & 84 & $16.00-887.00$ & 59 & $2.00-441.00$ & 0.08 \\
ALT & 54 & $14.00-420.00$ & 48 & $15.00-232.00$ & $0.01 *$ \\
Bilirubin & 3.2 & $0.80-61.00$ & 3 & $1.20-18.00$ & 0.3 \\
PT & 15.3 & $12.50-62.00$ & 15.2 & $12.40-58.00$ & 0.9 \\
INR & 1.62 & \pm .84 & 1.59 & \pm .74 & 0.7 \\
Na & 126 & $90.00-1112.00$ & 126 & $90.00-140.00$ & 0.2 \\
K & 4 & $2.30-32.00$ & 3.8 & $2.30-5.00$ & 0.6 \\
PMNL in ascitic fluid & 600 & $250.00-70000.00$ & 100 & $50.00-300.00$ & $<0.00 I^{* *}$ \\
\hline
\end{tabular}

Table 3 Classification of studied patients according to PMNL count of ascetic fluid

\begin{tabular}{lllll}
\hline & & No & $\%$ & P value \\
\hline Group I & SBP & 307 & $76.70 \%$ & $<0.00 I^{* *}$ \\
Group 2 & Non SBP & 93 & $23.30 \%$ & \\
& Total & 400 & $100 \%$ & \\
\hline
\end{tabular}

It was also found that positive cultures were gram negative in 77 patients culture $(60.2 \%)$ and gram positive in 51 patients culture $(39.8 \%)(\mathrm{p}<0.001)$ as shown in (Table 5). In the presnt study, the causative micro organism was found to be $E$ coli which was found in 57 patients (44\%), Staph aureus in 32 patients $(25 \%)$ and then $K$ pneumoniae found in 20 patients (16\%) finally Strept pneumoniae in 19 patients (15\%) of culture positive patients (Table 6).
It was also found that Gram negative bacteria were highly sensitive for Amoxicillin and Clavulanic acid (AMC), Meropenem and Erythromycin (Table 7) (Table 8) meanwhile Gram positive bacteria were highly sensitive for Amoxicillin and Clavulanic acid (AMC), Ofloxacin and cefoprazone (Table 7). So from results obtained In this study, it could be concluded that. Amoxicillin and Clavulanic acid (AMC) the most sensitive antibiotic to be used as an oral antibiotics 
giving $92 \%$ sensitivity on all the bacteria detected $(88.3 \%$ for Gram negative cultures and $98 \%$ for Gram positive cultures) (Table 9).

Table 4 Classification of SBP patients according to culture results

\begin{tabular}{lllll}
\hline & No & $\%$ & P value \\
\hline Culture & Negative (CNNA) & $17900 \%$ & $58.30 \%$ & $0.017^{*}$ \\
& Positive & 128 & $41.70 \%$ & \\
& Total & 307 & $100 \%$ & \\
\hline
\end{tabular}

Table 5 Classification of SBP according to gram stain

\begin{tabular}{llll}
\hline Organism & No & $\%$ & P \\
\hline Gram negative & 77 & $60.20 \%$ & $<0.00 I^{* *}$ \\
Gram positive & 51 & $39.80 \%$ & \\
Total & 128 & $100 \%$ & \\
\hline
\end{tabular}

Table 6 Types of micro-organisms in culture positive SBP

\begin{tabular}{llll}
\hline Bacteria in positive culture & No & $\%$ & P \\
\hline E coli (Gram -ve) & $5700.00 \%$ & $44 \%$ & $<0.00$ I** \\
Staph aureus (Gram +ve) & $3200.00 \%$ & $25 \%$ & \\
K Pnumonia (Gram -ve) & $2000.00 \%$ & $16 \%$ & \\
Strepto pnumonia (Gram +ve) & 19 & $15 \%$ & \\
Total & 128 & $100 \%$ & \\
\hline
\end{tabular}

P, Probability; Test used, Pearson's chi-squar; *, Significance <0.05; **, High significance

Table 7 Antibiotic sensitivity in positive cultures

\begin{tabular}{|c|c|c|c|c|c|c|c|}
\hline & \multicolumn{7}{|c|}{ Bacteria } \\
\hline & No & $\%$ & No & $\%$ & Total sensitive & $\%$ & $P$ value \\
\hline Cefatriaxone & 62 & $80.50 \%$ & 45 & $88.20 \%$ & 107 & $83.50 \%$ & - \\
\hline AMC (Amoxicillin and & 68 & $88.30 \%$ & 50 & $98.00 \%$ & 118 & $92.19 \%$ & 0.6 \\
\hline SAM(Ampicillin- & 64 & $83.10 \%$ & 48 & $94.10 \%$ & 112 & $87.50 \%$ & 0.8 \\
\hline \multicolumn{8}{|l|}{ Salbactam) } \\
\hline Ofloxacin & 62 & $80.50 \%$ & 50 & $98.00 \%$ & 112 & $87.50 \%$ & 0.8 \\
\hline Cefoprazone & 61 & $79.20 \%$ & 50 & $98.00 \%$ & III & $86.72 \%$ & 0.8 \\
\hline Clarithromycin & 63 & $81.80 \%$ & 45 & $88.20 \%$ & 108 & $84.38 \%$ & 0.96 \\
\hline Erythromycin & 67 & $87.00 \%$ & 40 & $78.40 \%$ & 107 & $83.60 \%$ & I \\
\hline Teicloplanin & 60 & $77.90 \%$ & 46 & $90.20 \%$ & 106 & $82.80 \%$ & 0.96 \\
\hline Nitrofurantion & 60 & $77.90 \%$ & 45 & $88.20 \%$ & 105 & $82.03 \%$ & 0.92 \\
\hline SXT(Sulphamethoxazole & 57 & $74.00 \%$ & 45 & $88.20 \%$ & 102 & $79.70 \%$ & 0.8 \\
\hline \multicolumn{8}{|l|}{ And Trimethoprim) } \\
\hline norfloxacin & 51 & $66.20 \%$ & 46 & $90.20 \%$ & 97 & $75.78 \%$ & 0.6 \\
\hline Amikacin & 53 & $68.80 \%$ & 6 & $11.80 \%$ & 59 & $46.10 \%$ & $0.003^{*}$ \\
\hline
\end{tabular}

P, Probability; Test used; Pearson's chi-square or fisher-exact test; *, significance; $<0.05^{* *}$, High significance; S, sensitive

Citation: Gharabawy SE, Mashad NE, Sheta TF, et al. Prevalence and microbiological features of spontaneous bacterial peritonitis in hospitalized ascitic patients: Single center study.J Bacteriol Mycol Open Access. 20I8;6(2):I60-I63. DOI: 10.15406/jbmoa.2018.06.00I96 


\section{Acknowledgements}

None.

\section{Conflict of interest}

The authors declare there is no conflict of interest.

\section{References}

1. Garcia-Tsao G. Bacterial infections in cirrhosis treatment and prophylaxis. J Hepatol. 2005;42(1):585-592.

2. Arroyo V, Jiminez W. Complications of Cirrhosis II. Renal and circulatory dysfunction. Lights and shadow of an important clinical problem. J Hepatol. 2000;32(1):157-170.

3. Abeer MS, Hany ME, Nehal H, et al. Prevalence and characteristics of spontaneous bacterial peritonitis in hospitalized patients with ascites due to liver cirrhosis. Cairo University, Giza, Egypt; 2008.
4. Cho JH, Park KH, Kim SH, et al. Bacteremia is a prognostic factor for poor outcome in spontaneous bacterial peritonitis. Scand J Infect Dis. 2007;39(8):697-702.

5. Kang CI, KimSH, Park WB, et al. Clinical outcome of bacteremic spontaneous bacterial peritonitis due to extended-spectrum betalactamase- producing escherichia coliand klebsiella pneumonia. Korean J Intern Med. 2004;19(3):160-164.

6. Barreales M, Fernandez I. Spontaneous bacterial peritonitis. Rev Esp Enferm Dig. 2011;103:255-263.

7. Gills A, Singh A, Matreja PS, et al. Spontaneous bacterial peritonitis in liver chirrhosis: A Indian perspective. Euroasian J Hepato Gastroenterol. 2012;2(1):14-19.

8. Payal HP, Saklainhaider S, Kairavi JD, et al. A study of bacteriological profile of ascitic fluid in suspected clinical cases of spontaneous bacterial peritonitis at a tertiary care hospital in India. International Journal of Medical Science and Public Health. 2015;4(4):496-501. 